\title{
Genetic mechanisms associated with determinate growth habit in cowpea (Vigna unguiculata (L.) Walp.)
}

\author{
Krylova E.A. ${ }^{1 *}$, Burlyaeva M.O. ${ }^{1}$, Khlestkina E.K. ${ }^{1,2}$ \\ ${ }^{1}$ N.I. Vavilov All-Russian Institute of Plant Genetic Resources, St. Petersburg, Russia \\ ${ }^{2}$ Institute of Cytology and Genetics, SB RAS, Novosibirsk, Russia \\ *e-mail:e.krylova@vir.nw.ru
}

Key words: determinant growth habit, cowpea, Vigna unguiculata, terminal flower

Motivation and Aim: Cowpea (Vigna unguiculata (L.) Walp.) is an important grain legume crop. It is known two types of growth habit that are classified as determinate and indeterminate. In wild species the growth habit is indeterminate and terminal shoot meristem of these plants remains in a vegetative state throughout the production of vegetative and reproductive structures. In plants with determinant growth habit, terminal shoot meristem switches from vegetative to reproductive state. It is known that TFL1 is gene controlling determinate growth habit in many legume species. The aim of the present study was (1) to identify and characterize TFL1 homologs in Vigna genome sequence and (2) to reveal allelic diversity of TFL1 gene in V. unguiculata accessions with type of growth habit, sensitive and insensitive for different (dry and wet) environment conditions.

Methods and Algorithms: Based on the allelic differences of the TFL1 gene described in the literature, DNA markers for distinguishing genotypes with different growth habit were developed. The homologous sequences of TFL1 gene have been identified in Vigna Genome Server ('VigGS', http://viggs.dna.affrc.go.jp) using BLAST search. In silico analysis was performed with MEGA, FGENESH+ and PLACE software.

Results: Additional copies of the TFL1 gene were found in Vigna genome. Phylogenetic analysis allowed to establish that the duplications occur in the common ancestor of legumes. 12 combinations of DNA markers were developed based on the known mutations of the TFL1 gene and used for re-sequencing this gene in $V$. unguiculata accessions, sensitive and insensitive to changing type of growth habit in different environment conditions.

Conclusion: Comparison of $V$. unguiculata accessions with type of growth habit, sensitive and insensitive for different (dry and wet) environment conditions, suggest more complicate regulation of this trait than mechanism based on just TFL1 allelic differences. Further comparative transcriptomic studies are needed. 